\title{
The Predicted Dielectric Constant of an Amorphous PVDF Changing with Temperature by Molecular Dynamics Simulations
}

\author{
Taotao $\mathrm{Hu}^{1, *}$, Bingbing $\mathrm{Hu}^{2, *}$ and Yabin $\mathrm{Yan}^{3, *}$ \\ ${ }^{1}$ School of Highway, Chang'an University, Xi'an 710064, P. R. China. \\ ${ }^{2}$ Faculty of Printing and Packing Engineering, Xi'an University of Technology, Xi' an 710048, P. R. \\ China \\ ${ }^{3}$ Institute of Systems Engineering, China Academy of Engineering Physics, Chengdu 621900, P. R. \\ China \\ *E-mail: hutaotao@163.com, hubb416@xaut.edu.cn, yanyabin@gmail.com
}

doi: $10.20964 / 2018.11 .01$

Received: 2 July 2017 / Accepted: 14 August 2018 / Published: 1 October 2018

\begin{abstract}
PVDF, a soft dielectric material, has attracted great attention in the research field of soft smart materials. However, some of the mechanisms for the dielectric and piezoelectric behaviors of PVDF are still not clear. In this work, the temperature dependent dielectric constant of the amorphous PVDF is predicted using molecular dynamics (MD) simulations. By inspecting the change of density with respect to temperature, the glass transition temperature $\left(T_{g}\right)$ of the amorphous PVDF is successfully determined. To calculate the dielectric constant of PVDF, a constant electric field is applied to the simulation model at different temperatures. It is found that the applied electric field leads to the appearance of dipoles rotation. By increasing the strength of applied electric field, more dipoles rotate either along or against the direction of applied electric field. These dipoles rotation further results in the variation of polarization of PVDF. On the other hand, the dielectric constant is evaluated at different temperatures. The temperature is found to significantly affect the dielectric constant, and $\mathrm{T}_{\mathrm{g}}$ plays a critical role as the critical temperature for material's dielectric constant. Moreover, the increment of the dielectric constant becomes much slower above $\mathrm{T}_{\mathrm{g}}$. This study explains the mechanism for the dielectric behavior of amorphous PVDF and provides a clue to the future study of other related properties of polymers, such as, piezoelectricity and flexoelectricity.
\end{abstract}

Keywords: PVDF; Polarization; Atomistic model; Dielectric constant

FULL TEXT 
(C) 2018 The Authors. Published by ESG (www.electrochemsci.org). This article is an open access article distributed under the terms and conditions of the Creative Commons Attribution license (http://creativecommons.org/licenses/by/4.0/). 